\title{
The genetic architecture of low-temperature adaptation in the wine yeast Saccharomyces cerevisiae
}

\author{
Estéfani García-Ríos ${ }^{1}$, Miguel Morard ${ }^{1,2}$, Leopold Parts ${ }^{3,5}$, Gianni Liti ${ }^{4}$ and José M. Guillamón ${ }^{1 *}$
}

\begin{abstract}
Background: Low-temperature growth and fermentation of wine yeast can enhance wine aroma and make them highly desirable traits for the industry. Elucidating response to cold in Saccharomyces cerevisiae is, therefore, of paramount importance to select or genetically improve new wine strains. As most enological traits of industrial importance in yeasts, adaptation to low temperature is a polygenic trait regulated by many interacting loci.

Results: In order to unravel the genetic determinants of low-temperature fermentation, we mapped quantitative trait loci (QTLs) by bulk segregant analyses in the F13 offspring of two Saccharomyces cerevisiae industrial strains with divergent performance at low temperature. We detected four genomic regions involved in the adaptation at low temperature, three of them located in the subtelomeric regions (chromosomes XIII, XV and XVI) and one in the chromosome XIV. The QTL analysis revealed that subtelomeric regions play a key role in defining individual variation, which emphasizes the importance of these regions' adaptive nature.

Conclusions: The reciprocal hemizygosity analysis (RHA), run to validate the genes involved in low-temperature fermentation, showed that genetic variation in mitochondrial proteins, maintenance of correct asymmetry and distribution of phospholipid in the plasma membrane are key determinants of low-temperature adaptation.
\end{abstract}

Keywords: Quantitative trait loci, Cold adaptation, Industrial yeast, Subtelomeres, Lipid asymmetry, Reciprocal hemizygosity analysis

\section{Background}

Low temperature is one of the most important environmental stresses that influences the life and distribution of living organisms. In the yeast Saccharomyces cerevisiae, reductions in environmental temperature have widespread effects on growth and survival. At low, but permissive, temperatures $\left(10-18{ }^{\circ} \mathrm{C}\right)$, metabolic activity and growth rates lower. This is relevant for the industrial exploitation of yeast since brewing and some wine fermentations take place at around $12-15{ }^{\circ} \mathrm{C}$. Low temperatures are used in wine fermentations to enhance production and to retain flavor volatiles, which enable the production of white and "rosé" wines with greater aromatic complexity $[1,2]$.

\footnotetext{
* Correspondence: guillamon@iata.csic.es

${ }^{1}$ Departamento de Biotecnología de los alimentos, Instituto de Agroquímica

y Tecnología de los Alimentos (CSIC), Avda. Agustín Escardino, 7,

E-46980-Paterna, Valencia, Spain

Full list of author information is available at the end of the article
}

Yeast undergoes considerable stress during wine fermentation from high concentrations of sugars in grape must, which leads to high osmotic pressure at the beginning of the process. As fermentation proceeds, ethanol accumulation, limiting nitrogen concentration, or even the presence of $\mathrm{SO}_{2}$, impose further pressure. In addition to these inherent difficulties to the process, temperatures below the optimum range of growth (around $32{ }^{\circ} \mathrm{C}$ ) [3] affect yeast growth and fermentation rates, and give rise to both a prolonged lag phase and the production of stuck and sluggish fermentations [4].

Changes in gene expression levels, ploidy and copy number variation (CNV) serve as the main genetic adaptive signatures of wine yeasts to fermentative processes [5]. Other enological traits, such as ethanol production, residual sugar after fermentation, nitrogen uptake and volatile acidity, are complex traits determined by multiple quantitative trait loci (QTL) [6]. The genetic mechanisms that underlie their variation can be identified by a linkage analysis. This 
approach uses crosses between two phenotypically different strains, and searches for a statistical link between the phenotype and genetic markers of segregant strains $[7,8]$. QTL mapping has been successfully applied to hightemperature growth [9-11], sporulation [12-15], cell morphology [16], drug sensitivity [17], ethanol tolerance and growth $[15,18,19]$, protein abundance $[20,21]$, and flocculation [22, 23]. QTL approaches have also been used to dissect the molecular basis of several wine yeast metabolic traits, such as acetic acid production, hydrogen sulfide production, and for the release of volatile phenol [24], nitrogen utilization and metabolite production [25], and the production of acetic acid, glycerol, and residual sugar concentrations [26].

To identify the genetic variants that affect lowtemperature adaptation in wine yeast, we performed QTL mapping using a set of segregants derived from a cross between two industrial wine yeast strains with a divergent phenotype at low temperature [27], but with a very similar genotype. We identified four genomic regions located in the different chromosomes implicated in the fermentation process at low temperature in industrial wine yeast, and ran a reciprocal hemizygosity $(\mathrm{RH})$ analysis for validation purposes. We identified subtelomeres to be an important source of genetic variation in industrial yeast [28], and found both the mitochondria and proteins involved in maintaining correct asymmetry and distribution of phospholipid in the plasma membrane played a crucial role in low-temperature growth.

\section{Results}

\section{Genetic characterization of parental strains}

We investigated the genetic basis of low-temperature adaptation in wine yeast in two $S$. cerevisiae enological strains, P5 and P24, characterized in a previous study [27], as displaying extreme differences in fermentation ability at $15{ }^{\circ} \mathrm{C}$. P5 corresponds to commercial strain Lalvin ${ }^{\circ} \mathrm{ICV}$ GRE, which is marketed for temperature fermentations ranging from 15 to $30{ }^{\circ} \mathrm{C}$. P24 has no commercial name since it is undergoing its development stage. The genomes of these two wine strains were sequenced and compared with that of reference strain S288c. We used seven informative genes to classify the parental strain [29] to construct a maximum likelihood phylogenetic tree of 15 strains of different origins. Figure 1a shows the five clean lineage described by Liti et al. (2009) [30], which are well

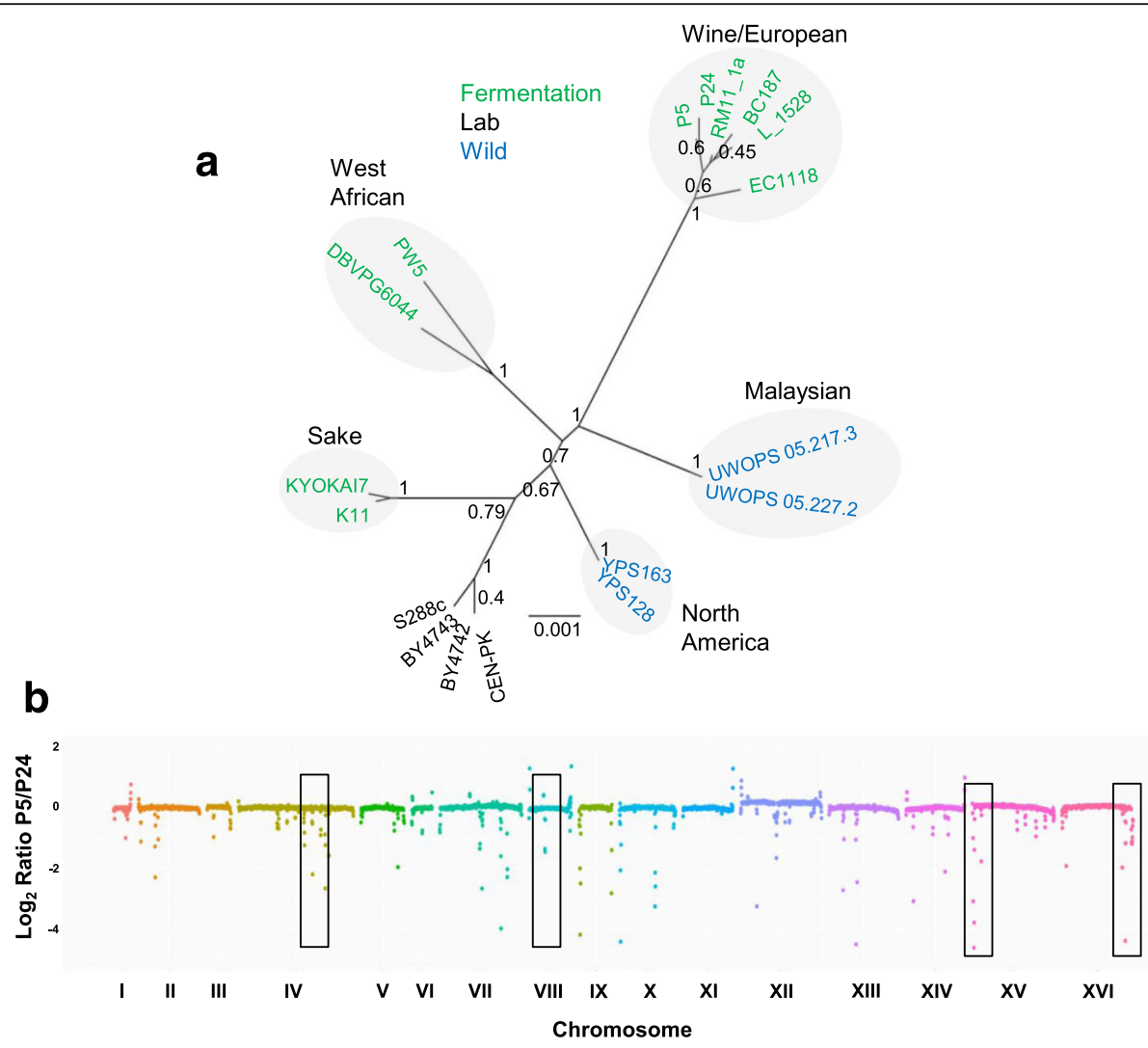

Fig. 1 Genomic and phylogenetic analyses of parental strains. a The evolutionary history was inferred using the ML method with 1000 replicates of the bootstrap test based on concatenated nucleotide sequences of seven phylogenetic informative loci. Different colors denote the strain's origin. b Copy number variation across the genome (chromosomes along $x$-axis and log2 ratio in $y$-axis). The four regions with a CNV bigger than $4 \mathrm{~kb}$ are marked in the figure 
clustered. As expected, both parental strains belonged to the Wine/European lineage with a bootstrap value of 1 .

Based on the raw sequence data, 43666 and $42983 \mathrm{mu}$ tations in strain P5 and strain P24, respectively, were identified compared to the reference strain. Of the total number of single-nucleotide polymorphism (SNPs), 36830 were common to both wine strains (Additional file 1: Table S1), with 6836 and 6153 specific mutations in P5 and P24 respectively, of which 86 and $82 \%$ were homozygous and representing a sequence divergence of 0.05\% (Additional file 2: Figure S1). Approximately 30\% of the unique SNPs of both wine strains were nonsynonymous changes in the coding region, which resulted in an amino acid change. This set of strain specific mutations was used for the linkage analysis.

Certain classes of variants, such as insertions and deletions (indels), are expected to have dramatic consequences for gene products, and therefore constitute particularly interesting candidates for contributing to phenotypic variation. The comparison with the reference strain yielded 867 and 874 strain specific indels, in which approximately 14 and 13\% were within the coding sequence, in the P5 and P24 respectively. When examining the distribution of these strain specific indels to each strain along chromosomes, some (chromosomes X, XI, XV and XVI) showed an enrichment of variants. Within open reading frames (ORFs), the indels with lengths that were multiples of three were highly enriched when compared with the noncoding sequence. This is consistent with strong purifying selection against frameshifts. Those indels in the coding sequence with lengths that were not multiples of three were located mainly in functionally uncharacterized genes. This confirmed that this group of genes was, on average, under lower purifying selection pressure [31].

We also detected the copy number variation of the genomic regions between both strains $(\mathrm{CNV})$. Ninety-three $\mathrm{CNV}$ longer than $900 \mathrm{bp}$ were detected across the genome comparison made between both strains. Figure $1 \mathrm{~b}$ shows the copy number variation ( $\mathrm{CNV}$ ) across chromosomes. Although there were some regions with variation, only four were larger than $4 \mathrm{~kb}$. These four regions were located in chromosome IV and in the subtelomeric regions of chromosomes VIII, XV and XVI. Most of these CNV were classified as transposable elements and subtelomeric regions. These results are in line with previous observations [31], which found very limited CNV in nonsubtelomeric regions and extensive variation in subtelomeric regions.

\section{Genetic and phenotypic characteristics of segregants}

For the QTL analysis, we generated populations of segregants by crossing the two wine strains of different cold tolerance for up to 13 generations (F13). This strategy allowed us to increase the resolution by reducing linkage between nearby QTLs. To study the genetic diversity of segregant populations, five differential SNPs of chromosome III in 30 segregants of F6 were genotyped. A recombination frequency of 0.39 was assessed, and there was an average of 10 haplotypes per SNP (data not shown). The F13 segregants were screened for their low temperature adaptation by calculating the maximum growth rate of each segregant and the two parental strains (Fig. 2a).

Transgression levels, that is, the percentage of segregants that exceed the phenotypic range of their parents by at least 2 standard deviations [24], can provide insight into the genetics that underlies complex traits. Sixteen percent of transgressive segregants better performed if compared with the superior parental, while just $0.66 \%$ of segregants presented a lower transgressive value than the inferior strain. Trait heritability was over $65 \%$, which indicates the importance of genetic determinism under our conditions. An example of the growth curves of two transgressive segregants is offered in Fig. $2 b$.

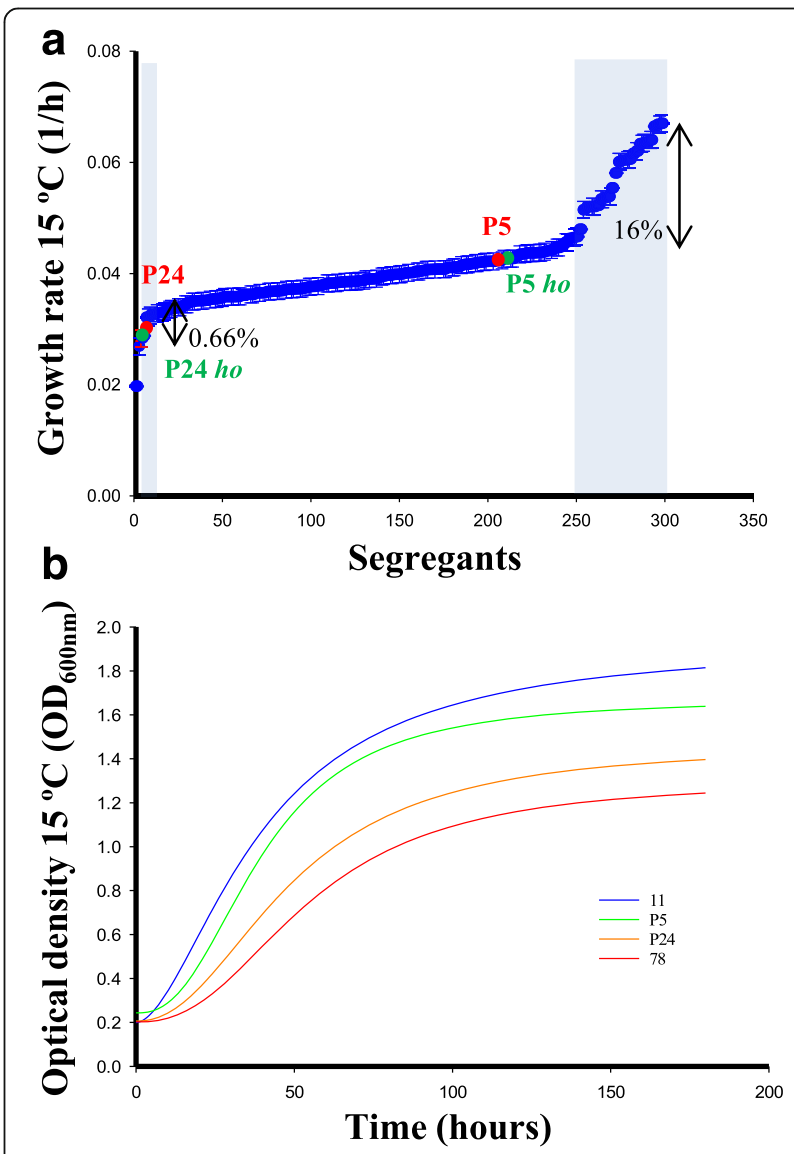

Fig. 2 Growth rate variation at low temperature. a Growth rate values at $15^{\circ} \mathrm{C}$ are shown on the $y$-axis for 300 ranked segregants. Blue indicates transgressive phenotypic space, and segregants with transgressive phenotypes (exceeding two parental standard deviations). Red dots indicate parental strains. b Detailed growth curves of parental and transgressive segregants (11 and 77) with extreme traits in the P5XP24 cross 


\section{Low-temperature selection of segregant populations}

The F13 diploid population was subjected to a selection experiment at 15 and $28{ }^{\circ} \mathrm{C}$ for approximately 50 generations ( 8 rounds of batch serial dilution; Additional file 3 : Figure S2). Figure 3 shows the improvement in the growth rate $\left(\mathrm{h}^{-1}\right)$ of the selected population (SP) compared with the F13 population. At low temperature, the growth rate of the selected population improved in both yeast extract peptone dextrose (YPD) (97\%, Fig. 3a) and synthetic must (SM) (66\%, Fig. 3c). The control population, which was cultured at $28{ }^{\circ} \mathrm{C}$, was also phenotyped in both media. The YPD selected population at $28{ }^{\circ} \mathrm{C}$ showed an improved growth rate (57\%, Fig. 3b). No significant differences were obtained for the SM selected population at $28^{\circ} \mathrm{C}$ (Fig. 3d).

To study if the improved growth rate in populations was due to either the temperature selective pressure or an unselective stress, each set was exposed to the opposite temperature to which they were selected. This meant that the SP at $15^{\circ} \mathrm{C}$ was grown at $28^{\circ} \mathrm{C}$ and that selected at $28^{\circ}$ $\mathrm{C}$ was grown at $15{ }^{\circ} \mathrm{C}$, and their growth rates were also compared with the F13 population (Additional file 4: Figure S3). As expected, in YPD medium, the selected population either at 28 or $15{ }^{\circ} \mathrm{C}$ didn't improve its growth or grew worse when was cultivated to the opposite temperature, revealing a selection based on the temperature. However, the selected population in SM also improved its growth when was cultivated in a non-selective temperature, indicating that this complex medium also exerted a strong pressure during the selection.

\section{Allele frequency analysis reveals that four QTLs are related to low-temperature adaptation during alcoholic fermentation}

A pool of the selected populations at $15{ }^{\circ} \mathrm{C}$ in YPD and SM and at $28{ }^{\circ} \mathrm{C}$ in $\mathrm{SM}$ were whole-genome sequenced and compared with the whole-genome sequence of a pool of the F13 population before selective growth at both temperatures. The allele frequency analysis allowed us to map the QTL intervals responsible for phenotypic variation. Four distinct QTLs for the three assayed conditions $\left(15{ }^{\circ} \mathrm{C}\right.$ in YPD, $15^{\circ} \mathrm{C}$ in SM and $28^{\circ} \mathrm{C}$ in SM) were found (Fig. 4 and Additional file 5: Figure S4). QTLs were detected for $28^{\circ} \mathrm{C}$ (orange triangles) on chr I, for $15{ }^{\circ} \mathrm{C}$ in YPD (red triangles) on chr XVI and for $15{ }^{\circ} \mathrm{C}$ in SM (green triangles) on chr XIII, XIV, XV and XVI. The QTL found at low temperature in YPD overlapped with that located in chromosome XVI at low temperature in SM. This finding suggested that this region could be important for low-temperature adaptation, and independently of media. Except for the QTL located in chromosome XIV, all the others were located in the subtelomeric regions. These results reinforce the hypothesis that
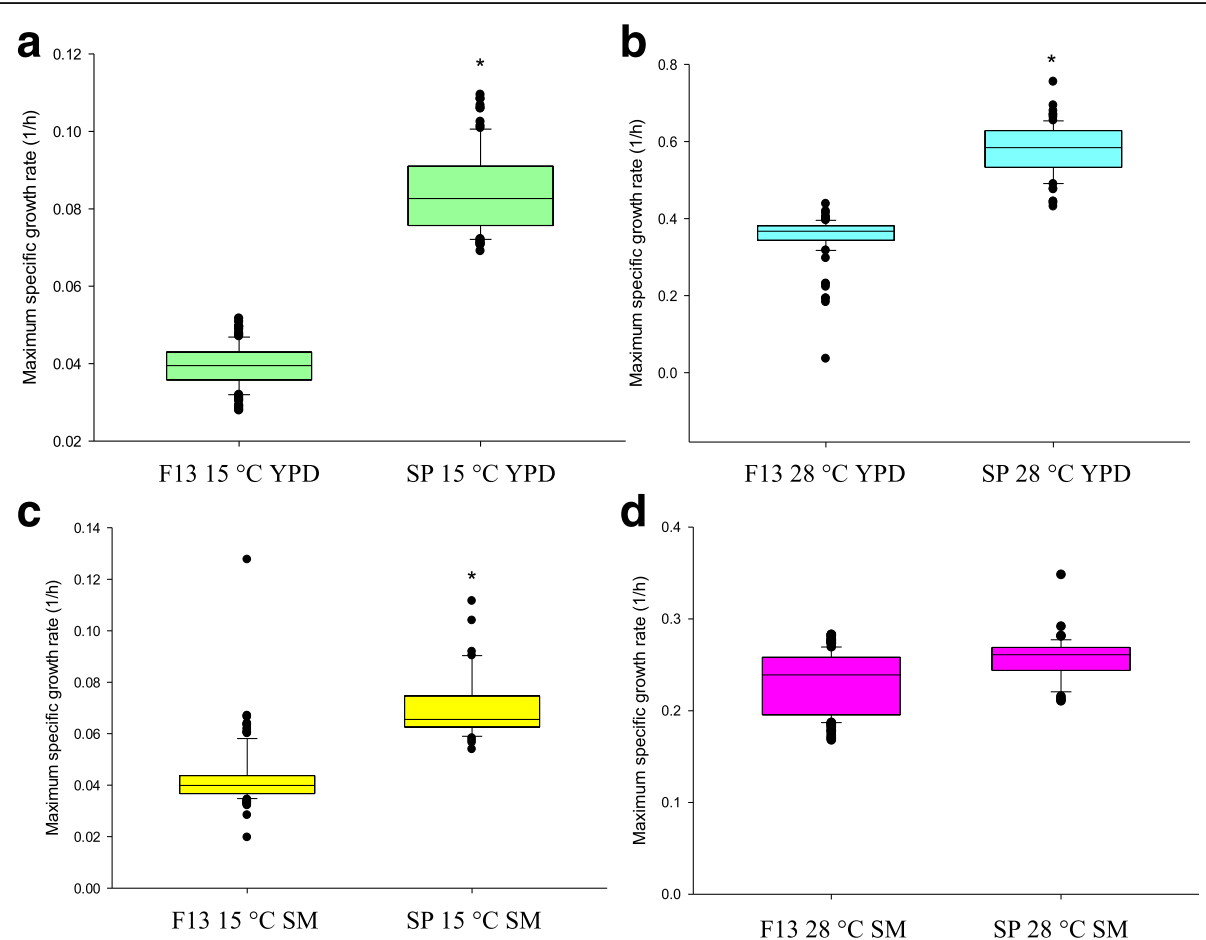

Fig. 3 Phenotyping (growth rate) of the hybrid population after the selection experiment compared with the unselected F13 population. Selected population (SP) in the YPD medium (a) and synthetic must (SM) (c) at $15^{\circ} \mathrm{C}$. Selected population (SP) in YPD (b) and SM (d) at $28{ }^{\circ} \mathrm{C}$. Box plot represents $\mu$ max distribution in each population and the black bar inside the box represents the mean value. *Significant differences in the SP compared with the F13 


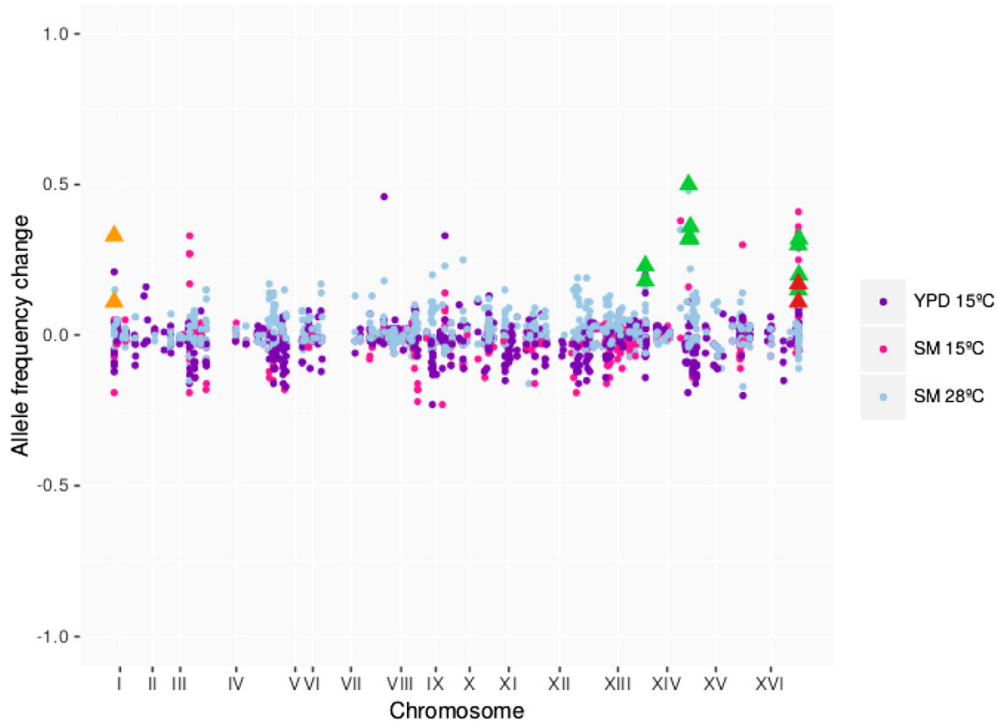

Fig. 4 QTL analysis for low-temperature adaptation. Genomic DNA samples were extracted from an unselected pool (F13) and three pools of segregants were selected at $15^{\circ} \mathrm{C}$ (YPD and SM) and $28^{\circ} \mathrm{C}$ (SM). The figure shows the allele frequency change of the three selected pools compared with the unselected population. QTLs are indicated at the corresponding positions with orange triangles $\left(28^{\circ} \mathrm{C} \mathrm{SM}\right)$, red triangles $\left(15^{\circ} \mathrm{C} \mathrm{YPD}\right)$ and green triangles $\left(15^{\circ} \mathrm{C} \mathrm{SM}\right)$

subtelomeric regions are a major source of the divergence of genome sequences and gene content in S. cerevisiae, and directly contribute to strain-specific adaptation processes.

\section{Validation of the QTLs detected in subtelomeric regions by reciprocal hemizygosity $(\mathrm{RH})$ analyses}

Subtelomeric regions are difficult to sequence and assemble due to their wide variation, duplication levels and shared homologies between different chromosome ends. Thus subtelomeres are generally incomplete in most genome projects, which precludes the identification of causative genes of a trait in these regions [32]. To validate the involvement of subtelomeric regions as QTLs of lowtemperature adaptation, two hemizygous diploid P5/P24 hybrid strains were constructed, which retained a single copy of the subtelomere from either the superior (P5) or inferior parent (P24), while the other copy was deleted. As growth rate and fermentation activity are directly correlated in these strains [27], they were tested during lowtemperature fermentations to estimate the phenotypic differences between the two reciprocal hemizygotes of each subtelomere. Fermentation activity was estimated by calculating the time required to ferment $100 \%$ (T100) of the sugars in the SM at 15 and $28{ }^{\circ} \mathrm{C}$ and it also is an interesting trait for our aim of detecting genome regions involved in adaptation to low temperature fermentations. The fermentation activity of the hemizygous strains is presented in Fig. 5 as the relative T100 compared with hybrid P5/P24. The parental origin of the subtelomere produced an opposite impact on fermentation activity in most cases. Thus the absence of the P5 right XVI-subtelomere caused a long delay of the end of fermentation at $28{ }^{\circ} \mathrm{C}$ and the inability to finish it at a low temperature. Conversely, lack of the same region that belonged to P24 significantly ( $p$-value $\leq 0.05$ ) improved fermentation at low temperature compared with the control hybrid strain P5/P24. Thus the P5 XVI-subtelomere must contain genes of paramount importance for fermentation activity in this strain, regardless of the temperature applied in the process. However, this same region in the P24 strain did not seem to be connected

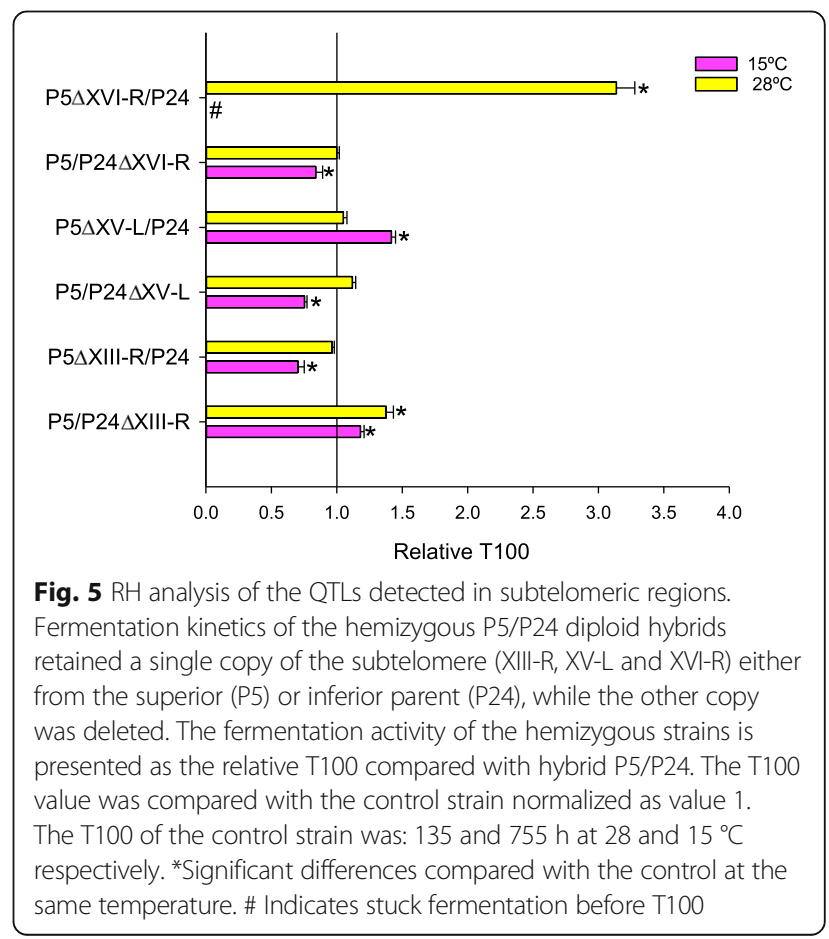


with temperature adaptation because its absence resulted in a haploproficient phenotype at low temperature.

Similarly, absence of the P24 right XIII-subtelomere caused a significant delay in the fermentation kinetics at both 15 and $28{ }^{\circ} \mathrm{C}$, whereas absence of the same region that belonged to P5 significantly improved fermentation at low temperature. The hemizygosity of the left XV-subtelomere was the only one to have an impact on fermentation activity at low temperature. Absence of this region in P5 significantly delayed the end of the fermentation at $15{ }^{\circ} \mathrm{C}$, whereas lack of the P24 region improved this fermentation activity at low temperature.

Identification of the causative genes of chromosome XIV QTL The QTL located in chromosome XIV was the only one not to be located in a subtelomeric region. The four genes (AGA1, COQ2, FPK1 and PET494) closest to the mapped QTL were selected for carrying out reciprocal hemizygosity analyses (Fig. 6a). MVD1 and TRM112, also in this region, were not selected because they are essential genes in the background of BY4741 strains. AGA1 is an agglutinin involved in sexual reproduction whose deletion has no effect on fermentation activity. However, the hemizygous hybrid strains of the other three genes clearly differed in fermentation activity at low temperature (Fig. 6b).

PET494 and COQ2 are mitochondrial proteins whose presence in hemizygosity produces impaired fermentation activity in both strains and at both temperatures, but mainly at a low temperature. PET494 is a translational activator of one of the subunits (COX3) of cytochrome $\mathrm{c}$ oxidase [33] and COQ2 encodes a transferase that catalyzes the second step in ubiquinone (coenzyme Q) biosynthesis [34]. The deletion of both genes provokes the absence of respiratory growth.

Finally, FPK1 is a Ser/Thr protein kinase that phosphorylates several aminophospholipid translocase family members (flippases) by regulating phospholipid translocation and membrane asymmetry. The reciprocal hemizygote that carries the P5 allele has no effect on the fermentation kinetics at any temperature. However, the hemizygous strain that carries the P24 allele caused a substantial delay $(\sim 340 \mathrm{~h})$ in low-temperature fermentation. The impaired fermentation activity of P5 $\Delta f p k 1 / \mathrm{P} 24$ was also confirmed by a spot assay (Fig. 6c), which also showed an important growth defect of this hemizygous strain at low temperature. P24 has a substitution $\mathrm{R} 520 \mathrm{~K}$ in this gene that could be the cause of the inferior phenotype of this strain at low temperature.

\section{$\mathrm{RH}$ analysis of wine-lab strain hybrids for the presumptive individual genes contained in the QTL subtelomeric regions}

The truncation of subtelomeric regions involved the deletion of some genes not connected with low temperature. As we have information only on the genes present in the first sequenced strain (S288c), in which special efforts were made to clone and sequence each telomere region,

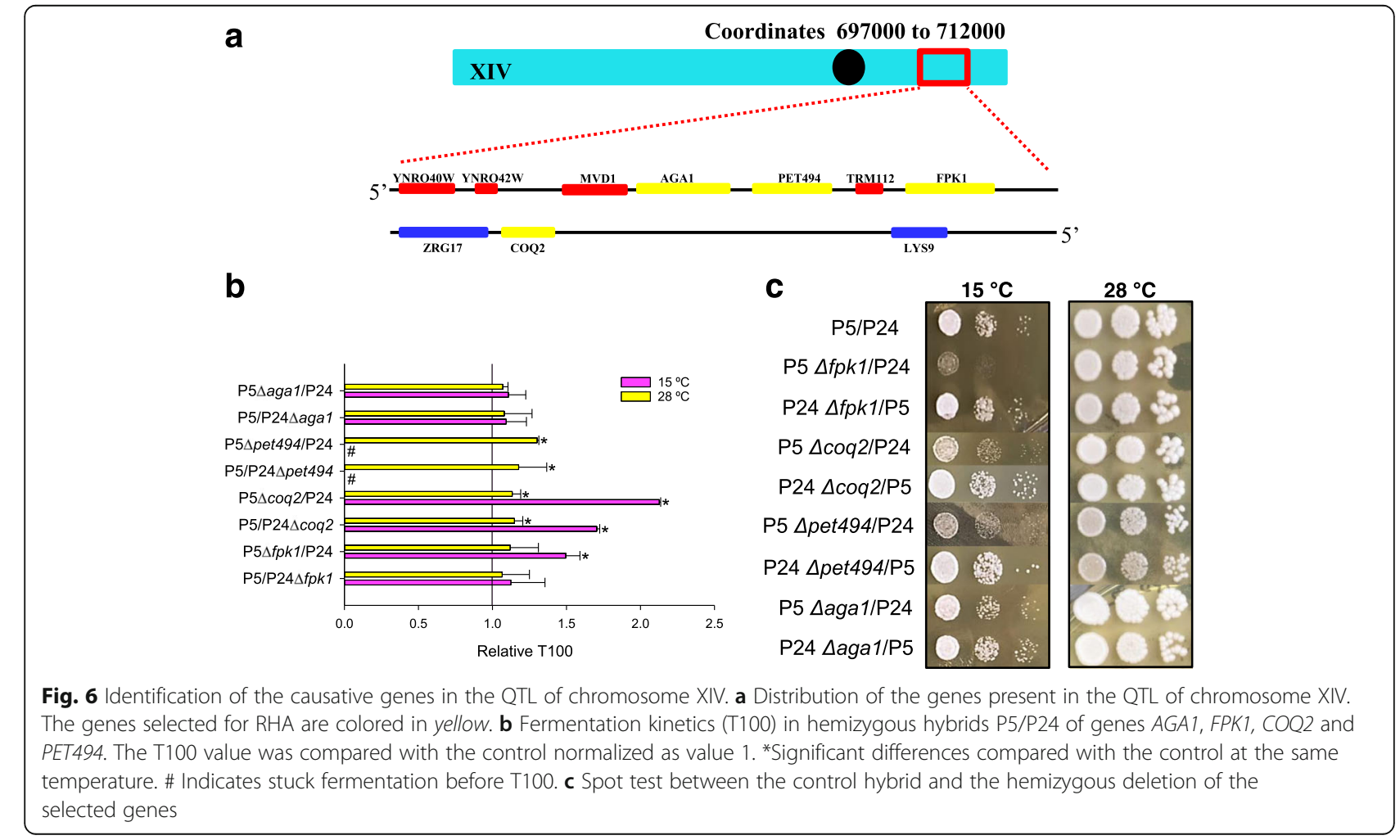

Coordinates 697000 to 712000 
we used the sequence available in the Saccharomyces Genome Database (SGD: http://www.yeastgenome.org) to perform a large-scale $\mathrm{RH}$ analysis with the genes known to be present in subtelomeric regions $(\sim 20-30 \mathrm{~Kb})$, and those that are not essential (Additional file 6: Table S2) [35]. The haploid single gene deletion strains in the background of the BY4741 lab strain were crossed with the stable haploids of each parental wine strain by constructing hemizygous hybrid lab/wine strains (BY4741/P5 and BY4741/P24). The fermentation capacity of the resulting hemizygotes, which contained only the wine strain allele of each individual candidate gene, were tested on SM at 15 and $28^{\circ} \mathrm{C}$ and compared to the corresponding capacity of the lab/wine hybrid with both parental alleles intact (Fig. 7). This strategy allowed us to compare the fitness of the different wine alleles and to attempt to discriminate the contribution made by each gene to the phenotype.

The RH analysis of individual genes showed both haploinsufficient and haploproficient strains in low-temperature fermentation activity. Haploproficiency meant that only the retention of the wine allele sufficed to improve the fermentation fitness of this hemizygous strain. Most haploproficient strains retained the P5 allele, which reinforces the superior fitness of this strain to grow and ferment at low temperature. Conversely, haploinsufficiency denoted a major function in low-temperature adaptation because a drop in the gene-dose impaired the fitness of the hemizygous strain. Most of these hemizygous strains had either a minimal or null impact on fermentation activity at $28{ }^{\circ} \mathrm{C}$ whereas they were severely affected at low temperature. At $15{ }^{\circ} \mathrm{C}$, the hemizygosity in most of the tested genes produced haploinsuffiency in the hybrid of both wine strains. Of the genes analyzed in the XVI-subtelomere region (Fig. 7d), QCR2, AQY1, YPR197c and ARR1 impaired only the low-temperature fermentation activity of the hemizygous P5/BY4741 strains, while genes OPT2, YPR195c and YPR196c affected this fermentation fitness at $15{ }^{\circ} \mathrm{C}$ in the hemizygous P24/BY4741 strains. QCR2 is also a component of the mitochondrial inner membrane electron transport chain and $A Q Y 1$ is a spore-specific aquaporin. Finally, OPT2 is an oligopeptide transporter with a described role in the maintenance of lipid asymmetry between the inner and outer leaflets of the plasma membrane.

The same RHA analysis was also performed with the genes contained in the nonsubtelomeric QTL detected in chromosome XIV (Fig. 7b). This analysis confirmed the relevance of the FPK1 allele in the superior fitness of the P5 strain because hemizygous P5/BY4741 showed better
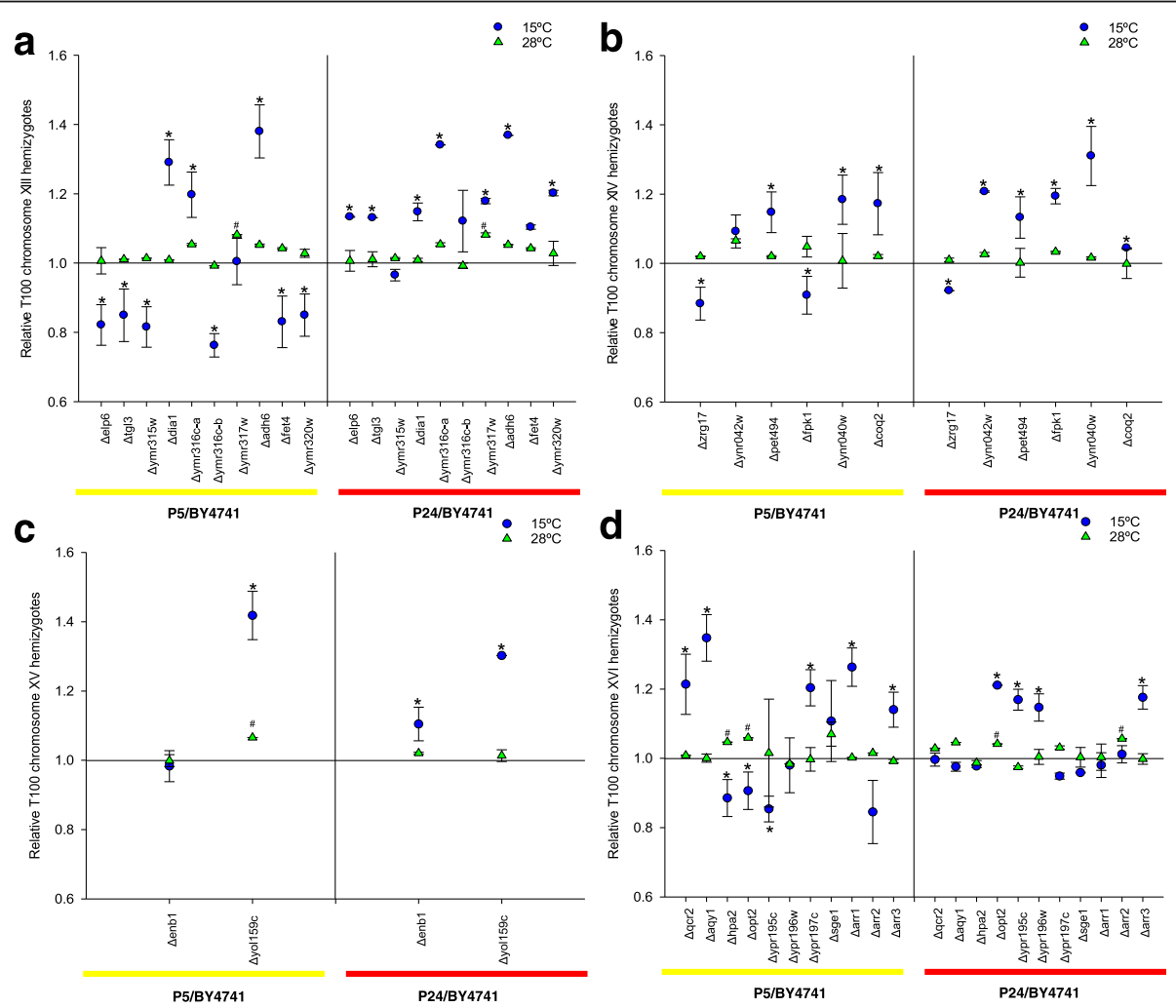

Fig. 7 RH analysis of the genes present in the QTL regions of chromosomes XIII (a), XIV (b), XV (c) and XVI (d). Hybrids were constructed using the mutants of the BY4741 collection and haploid parental strains. T100 is the time needed to consume the total amount of sugars present in must. The T100 value was compared with the control normalized as value 1. *Significant differences compared with the control at the same temperature 
fermentation activity, whereas hemizygous P24/BY4741 significantly delayed the end of fermentation. Furthermore, the importance of PET494 and COQ2 in low-temperature adaptation was also supported because both hemizygotes showed impaired fermentation activity at $15{ }^{\circ} \mathrm{C}$.

\section{Discussion}

Temperature had a strong effect on many life history traits, including growth, development and reproduction [36]. To elucidate the genetic basis that underlies low-temperature adaptation, we followed a method that began with a hybrid generated by crossing two strains with a divergent phenotype. This hybrid was used to generate segregating populations of a very large size, which were selected at environmental pressure (low temperature). Finally, QTL mapping was performed by identifying the regions of allelic enrichment by sequencing the segregant populations. This strategy has been previously applied to identify the QTLs responsible for heat tolerance in S. cerevisiae [9, 37, 38], but not to detect the genetic structure of low-temperature adaptation. Our study has also some particularities in that it makes it different to other similar approaches.

Most QTL analyses use strains that are highly divergent with a high density of segregating sites. Liti et al., (2009) reported the presence of five genetically diverged clean lineages in S. cerevisiae (populations that do not interbreed) and Parts et al., (2011) stated that the strains which belonged to clean lineages are ideal for linkage analyses as they have an even distribution of segregating sites across the genome, and these polymorphisms have coevolved in a specific genomic context. However, two strains that belonged to the same lineage were crossed (Wine/European) and were very close phylogenetically, as evidenced by the whole-sequence analysis of both strains. No more than $15 \%$ of the SNPs detected in both strains compared with the reference S228c were private mutations. While the low density of segregant sites between both strains proved to be an added difficulty for the QTL analysis since information about neighboring sites could not be used for more accurate allele frequency inferences, the strategy of intercrosses of segregants during the multiple generations that broke up linkage groups allowed individual QTLs to be determined and reduced to small numbers of variants [32,37].

Another different feature of our study compared with the commonly used bulk segregant analyses was the individual characterization of the 300 F13 segregants at their growth rate at low temperature. Segregants exhibited a wide range of cold tolerance phenotypes, but also a high percentage of these segregants displayed heterosis (hybrid vigor). This result revealed that the arrangement of alleles between both strains produced some combinations that improved fitness for it to become superior to parentals. This suggests that both strains contained alleles which contributed to the cold tolerance phenotype of these transgressive segregants.
However, this result was also very interesting for the industrial exploitation of these strains. The intercross during many generations of two industrial strains and the further selection of fitter genomic combinations by growth during several generations under selective pressure is a good genetic improvement method. These improved strains may be rapidly transferred and easily accepted by industry, which rejects the use of GMOs.

Our QTL analysis revealed the importance of the subtelomeric regions that contained a genetic variation responsible for traits of interest. Subtelomeric regions are generally incomplete in most genome projects. However, these regions cannot be ignored because, in yeast, $25 \%$ of all QTLs for many assessed traits map to beyond the last markers available. Yet the region contains only $8 \%$ of the genome [39]. Subtelomeric gene families evolve faster than their internal counterparts, and subtelomeric regions are more frequently sites of gene duplication [40], which suggests a unique role of subtelomeres as hotbeds for genomic evolution and innovation [41]. The whole-sequence analysis of parental strains also supports the idea that some of this variation might be due to the copy number of the genes present in different subtelomeres [32].

The hemizygotic truncation of one copy of the subtelomere in isogenic hybrid strain P5/P24 had antagonistic effects depending on the parental origin of this region. Thus the P5 right subtelomere of chromosome XVI must contain genes of much importance for wine fermentation, regardless of the temperature of this process. Conversely, the same region in P24 must not comprise these fermentative genes as some gene has a detrimental effect on the fermentation activity at low temperature. However, the most paradigmatic example of genomic regions that confer superior fitness to the P5 strain at low temperature is the left subtelomere of chromosome XV. The fermentation performance of the hemizygous strain that lacked the P5 copy was clearly affected at low temperature, but not at $28{ }^{\circ} \mathrm{C}$, and the hybrid strain that carried the P5 copy in hemizygotes significantly improved. Application of new long read sequencing technologies would reveal genes residing in these subtelomeric regions, and allow identifying the causal genes involved in adaptation at low temperature.

We detected only a nonsubtelomeric QTL in chromosome XIV. In this case, the RH analysis showed two mitochondrial proteins, which are essential for respiratory growth and extremely important in fermentation activity at low temperature. Although we may think that the absence of respiratory metabolism could not be important during a process with a dominant fermentative metabolism, it is well-known that the yeast strains which lack the mitochondrial function are sensitive to oxidative stress caused by reactive oxygen species (ROS) [42]. We recently proved that cells grown at low temperature are also subjected to stronger oxidative stress $[27,43]$. The $\mathrm{RH}$ analysis also 
showed that gene $F P K 1$ was very important for endowing better cold adaptation to P5 strain. This gene regulates flippase activity, which establishes plasma membrane asymmetry by flipping specific phospholipids from the extracellular to the cytosolic leaflet. The continuous remodeling of the phospholipid composition of cellular membranes as a response to low-temperature fermentations has been widely reported [44-47]. The implication of FPK1 in lowtemperature adaptation could be due to an improvement in the ability to maintain plasma membrane asymmetry. Finally, the RH analysis of the BY4741/wine hybrid strains revealed the key role during low-temperature fermentations of a gene (QCR2) that encodes a protein of the mitochondrial inner membrane and a gene (OPT2) involved in lipid asymmetry maintenance between the inner and outer leaflets of the plasma membrane, among others. This demonstrates the importance of both a fit mitochondria and the capacity of lipid remodeling in the plasma membrane for coping with low temperatures.

\section{Conclusions}

We followed a thorough strategy, previously described by Parts et al. (2011) [37], to elucidate the molecular basis that determines fitness or better adaptation during wine low-temperature fermentations. The QTL analysis and further RH analyses of the detected QTLs proved the importance of subtelomeres as a source of variation in industrial yeast and the need to invest efforts in sequencing these regions with new sequencing technologies with long reads from single molecules. We detected the individual genes involved in adaptation at low temperature fermentation by highlighting the importance of a fit mitochondria, perhaps for coping with greater oxidative stress at low temperature, and the maintenance of correct asymmetry and phospholipid distribution in the plasma membrane. Altogether this information is very useful for industrial yeast exploitation because the mechanisms involved in cold adaptation can be used as important traits for future selections of industrial cryotolerant strains or for their genetic improvement.

\section{Methods}

\section{Strain selection and advanced intercross lines}

We selected two industrial wine strains, P5 and P24, as parent strains for their marked phenotypic growth differences at low temperature [27]. Both strains were kindly provided by Lallemand Inc. (France). We generated derivative strains that were stable haploid and auxotrophic for uracil (ho::KanMX4 ura3s and ho::NatMX4 ura3s). The selected haploids didn't show significant differences ( $p$-value $\leq 0.05)$ in the growth rate with the parental strains (diploids) both at 28 and $15{ }^{\circ} \mathrm{C}$. We replaced the LYS2 with URA3 gene in the P5 strain. Such gene replacement restores the ability of growth in the absence of uracil and makes it unable to grow without lysine. The two parental strains with opposite mating types (P5 mat $\alpha$ URA3+ and P24 mat a LYS2+) were crossed in complete media (YPD) and grown overnight. Patches were replica-plated in synthetic minimal media (SD) to select diploid F1 hybrids. Two F1 hybrid replicas were grown overnight (spread over a whole Petri dish) and replica-plated on KAc (potassium acetate 1\%, agar 2\%) at $30{ }^{\circ} \mathrm{C}$ to be sporulated for 10 days. Sporulation efficiency (\% of sporulating cells) was monitored until it reached $>90 \%$. The cells from the whole plate were carefully collected and resuspended in $0.5 \mathrm{~mL}$ of sterile water, treated with an equal amount of ether and vortexed for $10 \mathrm{~min}$ to selectively kill unsporulated cells [48]. Cells were washed 4 times in sterile water, resuspended in $900 \mu \mathrm{L}$ of sterile water and treated with $100 \mu \mathrm{L}$ of zymolase $\left(10 \mathrm{mg} \mathrm{mL}^{-1}\right)$ to remove the ascus. Cell mixtures were vortexed for 5 min to increase spore dispersion and inter-ascus mating.

Cells were plated at high density to begin a second round of mating and meiosis (F2). The presence of two distinct markers (LYS2 and URA3) at the same genomic position prevents them from co-segregating in haploid cells, and thus allows selection for diploid cells segregating at that locus. In order to confirm this system, we dissected 10 tetrads from the F6 pool, all of which had the correct 2:2 segregation of the LYS/URA markers. The sporulated pool was treated with zymolase and plated at high density to start the next intercross generation, as described in the paragraph above (Additional file 7: Figure S5). This process was repeated until the F13 population was achieved to generate a large pool of segregants for sensitive and high resolution QTL mapping [37].

\section{Media and growth conditions}

The growth media selected for the experiments were SD (Yeast Nitrogen Base (YNB, Difco) supplemented with $20 \mathrm{~g} \mathrm{~L}^{-1}$ of glucose as the carbon source), YPD (glucose $20 \mathrm{~g} \mathrm{~L}^{-1}$, peptone $20 \mathrm{~g} \mathrm{~L}^{-1}$, yeast extract $10 \mathrm{~g} \mathrm{~L}^{-1}$ ) and synthetic grape must (SM), which was derived from that described by Riou et al. (1997) [49]. The SM composition included $200 \mathrm{~g} \mathrm{~L}^{-1}$ of sugars $\left(100 \mathrm{~g} \mathrm{~L}^{-1}\right.$ glucose $+100 \mathrm{~g} \mathrm{~L}^{-1}$ fructose), $6 \mathrm{~g} \mathrm{~L}^{-1}$ malic acid, $6 \mathrm{~g} \mathrm{~L}^{-1}$ citric acid, $1.7 \mathrm{~g} \mathrm{~L}^{-1}$ YNB without ammonium and amino acids, anaerobic factors $\left(0.015 \mathrm{~g} \mathrm{~L}^{-1}\right.$ ergosterol, $0.005 \mathrm{~g} \mathrm{~L}^{-1}$ sodium oleate and $0.5 \mathrm{~mL} \mathrm{~L}^{-1}$ Tween 80 ) and $0.060 \mathrm{~g} \mathrm{~L}^{-1}$ potassium disulfite. The assimilable nitrogen source used was $0.3 \mathrm{~g} \mathrm{~N} \mathrm{~L}^{-1}$ (0.12 $\mathrm{g} \mathrm{N} \mathrm{L}^{-1}$ as ammonium chloride and $0.18 \mathrm{~g} \mathrm{~N} \mathrm{~L}^{-1}$ in an amino acid form; the proportion of each amino acid was administered as previously proposed by Riou et al. (1997) [49]. Growth was monitored at $600 \mathrm{~nm}$ in a SPECTROstar Omega instrument (BMG Labtech, Offenburg, Germany). Measurements were taken every $30 \mathrm{~min}$ for 4 days after 20s pre-shaking for all the experiments. At low temperatures 
$\left(15{ }^{\circ} \mathrm{C}\right)$ however, microplates had to be incubated outside the spectrophotometer and then placed inside before being measured (every $3 \mathrm{~h}$ for 10 days). Microplate wells were filled with the required volume of inoculum and $0.25 \mathrm{~mL}$ of medium to always ensure an initial OD of approximately 0.1 (inoculum level of about $10^{6}$ cells $\mathrm{mL}^{-1}$ ). Uninoculated wells for each experimental series were also included in the microplate to determine, and to therefore subtract, the noise signal. All the experiments were carried out at least in triplicate. Growth parameters were calculated from each treatment by directly fitting OD measurements versus time to the reparameterized Gompertz equation proposed by Zwietering et al. (1990) [50]:

$$
\left.\mathrm{y}=\mathrm{D} * \exp \left\{-\exp \left[\left(\left(\mu_{\max } * \mathrm{e}\right) / \mathrm{D}\right) *(\lambda-\mathrm{t})\right)+1\right]\right\}
$$

where $y=\ln \left(\mathrm{OD}_{\mathrm{t}} / \mathrm{OD}_{0}\right), \mathrm{OD}_{0}$ is the initial $\mathrm{OD}$ and $\mathrm{OD}_{\mathrm{t}}$ is the OD at time $t ; \mathrm{D}=\ln \left(\mathrm{OD}_{\mathrm{t}} / \mathrm{OD}_{0}\right)$ is the asymptotic maximum, $\mu_{\max }$ is the maximum specific growth rate (h ${ }^{-1}$ ), and $\lambda$ the lag phase period (h).

For the spot assays, the cells were grown on YPD at $28{ }^{\circ} \mathrm{C}$ to the stationary phase (OD600 4) were harvested by centrifugation, washed with sterile water, resuspended in sterile water to an OD $(600 \mathrm{~nm})$ value of 0.5 , and followed by serial dilution. From each dilution, $3.5 \mu \mathrm{L}$ were spotted onto YPD agar plates. Plates were incubated at 28 and $15{ }^{\circ} \mathrm{C}$ for 2 and 9 days, respectively.

\section{Selection experiment}

The pools of the population size of $10-100$ million cells (estimated by plating serial dilutions and colony forming units (CFU) counting) were collected from the sporulation media and treated with ether and zymolase, as described above. Spores were grown in complete media (YPD) and synthetic must (SM), and were incubated at either optimum temperature $\left(28^{\circ} \mathrm{C}\right)$ or low temperature $\left(15^{\circ} \mathrm{C}\right)$ until the stationary phase was reached. All the cells were carefully collected and resuspended in distilled water and a small volume (the volume required to inoculate at an optical density (OD) of 0.2 ) of the expanded culture was transferred to $60 \mathrm{~mL}$ of fresh medium. Culture growth was monitored by measuring absorbance at $600 \mathrm{~nm}$ every $24 \mathrm{~h}$ at $28^{\circ} \mathrm{C}$, and every $48 \mathrm{~h}$ at $15^{\circ} \mathrm{C}$. The number of generations was calculated by the equation: $n=\left(\log \mathrm{N}_{\mathrm{t}}-\log \right.$ $\mathrm{N}_{0}$ )/ $\log 2$, where $\mathrm{n}$ is the number of generations, $\mathrm{N}_{0}$ is the initial OD and $\mathrm{N}_{\mathrm{t}}$ is the OD at time $t$. The experiment was carried out 8 times, after which the selected populations were analyzed.

\section{Sequencing}

The genome sequencing of the selected populations was performed by 5500xl SOLiD sequencing. Genomic libraries were prepared following the manufacturer's standard instructions. Emulsion PCRs were performed using the
SOLiD $^{\text {ma }}$ EZ Bead ${ }^{\text {tm }}$ Systems. Sequencing was carried out by 75 nt single-end read exact call chemistry (ECC) following the manufacturer's standard protocols. The whole-genome sequences are deposited in the Sequence Read Archive (SRA) database (http://www.ncbi.nlm.nih.gov/sra/) and are available with access number SRP048919. Breseq (v0.27.1) [51] pipeline was used to first align the reads of the reference S288c genome (using Bowtie2 [52], and to identify SNPs and indels with a frequency cutoff of 0.2. CNV detection was performed using CNV-seq [53] with a window size of $121 \mathrm{nt}$ (with a minimum log2 fold of 0.6 and a minimum $p$-value of 0.001)). P5 was used as the "reference" genome and P24 as the "test genome". Only CNV larger than $900 \mathrm{bp}$ were considered for the analysis. The sequences of seven informative loci [29] from 15 strains that represented pure groups were downloaded from the SGD (http:// www.yeastgenome.org/) and the NCBI (https:// www.ncbi.nlm.nih.gov/) to perform the phylogenetic analysis. Each gene sequence was aligned using mafft (v7.221) [54] individually and then concatenated. The phylogenetic tree was constructed using a Maximum Likelihood Method (ML) with RAxML [55] with 100 bootstrap replicates. The distribution of the SNPs among the strains was visualized using Circos 0.69.2 (http://circos.ca).

\section{Linkage analysis}

We retained variants that were covered with at least 30 sequencing reads in both control and selection experiments, and were present at $30-70 \%$ allele frequency in the initial segregant population. This ensures that only confident segregating alleles are analyzed. We then calculated the allele frequency changes in both biological replicates for each retained site, and called QTLs the alleles with a frequency change of at least 0.1 in the same direction in both replicates.

\section{Validation of QTLs}

QTLs were validated by reciprocal hemizygosity using the URA3 gene as a selectable marker [56]. Briefly, the haploid versions of the parental strains (P5 Mat $\alpha$, ho::HygMX, ura3::KanMX or P24 Mat a, ho::NatMX, ura3::PhleoMX) were used to delete each target subtelomeric region or gene, and to construct all the possible combinations. After the deletions of the candidate regions, strains were crossed to generate the hybrid strains and selected in double drugs plates. Diploid hybrid strains were detected by the benomyl assay $[57,58]$ and confirmed by Mat locus PCR [59], and the deletions of the target genes were confirmed by PCR using specific primers. The uncharacterized single copy ORF $Y M R 317 W$ was used as a target to truncate the chromosome XIII right subtelomeric region, gene YOL159C was used to truncate the chromosome XV left subtelomeric region and gene $S G E 1$ was employed in the case of the 
chromosome XVI right subtelomeric region. To identify the contribution of the alleles present in these regions, 60 hemizygote hybrids were constructed, each resulting from a cross between a derivative haploid P5 or a P24 strain and BY4741 that lacked one of these genes. A heterozygote hybrid strain with the wild-type BY4741 was also constructed and used as a control.

\section{Fermentation conditions}

Fermentations were performed at 28 and $15{ }^{\circ} \mathrm{C}$, with continuous orbital shaking at $100 \mathrm{rpm}$. Fermentations were done in laboratory-scale fermenters using $100-\mathrm{mL}$ bottles filled with $60 \mathrm{~mL}$ of SM. Fermentations were monitored by the density of media $\left(\mathrm{g} \mathrm{L}^{-1}\right)$ using a densitometer (Densito 30PX, Mettler Toledo, Switzerland). Fermentations were considered complete when density reached $995 \mathrm{~g} \mathrm{~L} \mathrm{~L}^{-1}$. Yeast cell growth was determined by absorbance at $600 \mathrm{~nm}$ and by plating on YPD.

\section{Statistical analysis}

All the experiments were carried out at least in triplicate. Physiological data were analyzed with the Sigma Plot 12.5 software, and the results were expressed as mean and standard deviation. To evaluate statistical significance, two tailed $\mathrm{t}$-student tests were applied with a $p$-value of 0.05 . $P$-values were corrected for multiple testing by the Bonferroni test. Phenotypic data were fitted to the reparameterized Gompertz model by nonlinear least-squares fitting using the Gauss-Newton algorithm as implemented in the nls function in the R statistical software, v.3.0. Phenotype heritability $\mathrm{H}^{2}$ was calculated as previously described [60], i.e., $\mathrm{H}^{2}=\left(\left(\operatorname{Var}_{\text {seg }}-\operatorname{Var}_{\text {env }}\right) / \mathrm{Var}_{\text {seg }}\right) \times 100$, where $\operatorname{Var}_{\text {env }}$ is the pooled variance among parental measurements and $\mathrm{Var}_{\mathrm{seg}}$ is the variance among phenotype values for segregants. Transgressive segregation was defined as in $[24,60]$ by the number of segregants whose phenotype level lay at least $2 \sigma$ higher than the mean phenotype level of the higher parent, or was $2 \sigma$ lower than the mean phenotype level of the lower parent. $\sigma$ was the pooled standard deviation of parents.

\section{Additional files}

Additional file 1: Table S1. Genomic comparison among strains. Single nucleotide polymorphism (SNPS) population distribution. SNPs were classified according to genome localization and change in protein sequence (nonsynonymous variant). (XLSX 5469 kb)

Additional file 2: Figure S1. Distribution of private nonsynonymous SNPs in P5 and P24 compared to S288c. An external circle indicates P24 and an internal circle indicates P5. Homozygous changes are colored in green, while heterozygous changes are marked in red. (PDF 243 kb)

Additional file 3: Figure S2. Workflow of populations' selection and sequencing. Cells were grown in complete media (YPD) and synthetic must $(\mathrm{SM})$, and were incubated at either optimum temperature $\left(28^{\circ} \mathrm{C}\right)$ or low temperature $\left(15^{\circ} \mathrm{C}\right)$ until the stationary phase was reached. At this time, the volume required to inoculate at an OD of 0.2 was re-inoculated into $60 \mathrm{~mL}$ of fresh medium. The experiment was carried out 8 times after which the selected populations were analyzed and sequenced. (PDF $43 \mathrm{~kb}$ )

Additional file 4: Figure S3. Hybrid population phenotyping after the selection experiment compared with the unselected F13 population using the opposite temperature to that used during the selection process (nonspecific improvement). The selected population (SP) in the YPD medium (A) and synthetic must (SM) (C) at $15^{\circ} \mathrm{C}$. The selected population (SP) in YPD (B) and SM (D) at $28^{\circ} \mathrm{C}$. Box plot represents $\mu$ max distribution in each population and the black bar inside the box represents the mean value. *Significant differences in the SP compared with the F13. (PDF $66 \mathrm{~kb}$ )

Additional file 5: Figure S4. QTL analysis for low-temperature adaptation. The figure shows the allele frequency change of the selected pools at YPD $15{ }^{\circ} \mathrm{C}$ (purple), SM $15^{\circ} \mathrm{C}$ (pink) and SM $28{ }^{\circ} \mathrm{C}$ (blue) compared with the unselected population. QTLs are indicated at the corresponding positions with red (YPD $\left.15^{\circ} \mathrm{C}\right)$, green $\left(\mathrm{SM} 15^{\circ} \mathrm{C}\right)$ and orange triangles (SM $\left.28^{\circ} \mathrm{C}\right)$. (PDF $\left.70 \mathrm{~kb}\right)$

Additional file 6: Table S2. List of genes used in the $\mathrm{RH}$ analysis with the BY4741 strain that are present in the subtelomeric regions and are not essential. (XLSX $13 \mathrm{~kb}$ )

Additional file 7: Figure S5. Outline of the construction of advanced intercross lines. We carried out a strategy that forces yeast cells through multiple rounds of random mating and sporulation to create advanced intercross lines (AlLs). This step can improve genetic mapping in two ways: increasing resolution by reducing linkage and unlinking nearby QTLs. (PDF $168 \mathrm{~kb}$ )

\section{Abbreviations}

CFU: Colony forming units; CNV: Copy number variation; indels: Insertions and deletions; KAc: Potassium acetate; OD: Optical density; ORF: Open reading frame; QTL: Quantitative trait loci; RHA: Reciprocal hemizygosity analysis; ROS: Reactive oxygen species; SM: Synthetic must; SNP: Single-nucleotide polymorphism; SP: Selected population; YPD: Yeast extract peptone dextrose

\section{Acknowledgements}

We acknowledge support of the publication fee by the CSIC Open Access Publication Support Initiative through its Unit of Information Resources for Research (URICI)

\section{Funding}

This work has been financially supported from the Spanish Government through MINECO and FEDER funds (AGL2013-47300-C3-3-R and PCIN-2015143 grants) and from Generalitat Valenciana through PROMETEOII/2014/042 grant, awarded to JMG. This study has been carried out in the context of the European Project ERA-IB "YeastTempTation" EGR thanks the Spanish government for an FPI grant BES-2011-044498 and MM also thanks the Generalitat Valenciana for a VALi+d ACIF/2015/194 grant.

\section{Availability of data and materials}

The data set supporting the results of this article is available in the Sequence Read Archive (SRA) database repository SRP048919 (http://www.ncbi.nlm.nih.gov/sra/ ?term=SRP048919). The data set supporting the results of this article is included in the article (and its additional files).

\section{Authors' contributions}

EGR conducted the experiments, analyzed the data and wrote the manuscript. MM and LP analyzed the data. JMG and GL conceived the study, participated in the study design and wrote the manuscript. All the authors have read and approved the final manuscript.

\section{Competing interests}

The authors declare that they have no competing interests.

\section{Consent for publication}

Not applicable.

Ethics approval and consent to participate Not applicable. 


\section{Author details}

1Departamento de Biotecnología de los alimentos, Instituto de Agroquímica y Tecnología de los Alimentos (CSIC), Avda. Agustín Escardino, 7, E-46980-Paterna, Valencia, Spain. ${ }^{2}$ Departament de Genètica, Facultat de Ciències Biològiques, Universitat de València, Dr. Moliner, 50, E-46100 Burjassot, València, Spain. ${ }^{3}$ European Molecular Biology Laboratory, Meyerhofstrasse 1, Heidelberg 69117, Germany. ${ }^{4}$ Institute of Research on Cancer and Ageing of Nice (IRCAN), CNRS UMR 7284-INSERM U1081, Faculté de Médecine, Université de Nice Sophia Antipolis, Nice, France. ${ }^{5}$ Wellcome Trust Sanger Institute, Hinxton CB101SA, UK.

\section{Received: 27 September 2016 Accepted: 9 February 2017} Published online: 14 February 2017

\section{References}

1. Torija MJ, Rozés N, Poblet M, Guillamón JM, Mas A. Effects of fermentation temperature on the strain population of Saccharomyces cerevisiae. Int J Food Microbiol. 2003;80:47-53.

2. Beltran G, Torija MJ, Novo M, Ferrer NN, Poblet M, Guillamón JM, et al. Analysis of yeast populations during alcoholic fermentation: a six year follow-up study. Syst Appl Microbiol. 2002;25:287-93.

3. Salvadó Z, Arroyo-López FN, Guillamón JM, Salazar G, Querol A, Barrio E, et al. Temperature adaptation Markedly Determines evolution within the genus Saccharomyces. Appl Environ Microbiol. 2011;77:2292-302.

4. Bisson LF. Stuck and sluggish fermentations. Am J Enol Vitic. 1999;50:107-19.

5. Infante JJ, Dombek KM, Rebordinos L, Cantoral JM, Young ET. Genome-wide amplifications caused by chromosomal rearrangements play a major role in the adaptive evolution of natural yeast. Genetics. 2003;165:1745-59.

6. Marullo P, Bely M, Masneuf-Pomarede I, Aigle M, Dubourdieu D. Inheritable nature of enological quantitative traits is demonstrated by meiotic segregation of industrial wine yeast strains. FEMS Yeast Res. 2004;4:711-9.

7. Mackay TFC, Stone EA, Ayroles JF. The genetics of quantitative traits: challenges and prospects. Nat Rev Genet. 2009;10:565-77.

8. Parts L. Genome-wide mapping of cellular traits using yeast. Yeast. 2014;31: 197-205.

9. Yang Y, Foulquié-Moreno MR, Clement L, Erdei É, Tanghe A, Schaerlaekens $K$, et al. QTL analysis of high thermotolerance with superior and downgraded parental yeast strains reveals new minor QTLs and converges on novel causative alleles involved in RNA processing. PLoS Genet. 2013;9: e1003693.

10. Sinha H, David L, Pascon RC, Clauder-Münster S, Krishnakumar S, Nguyen M, et al. Sequential elimination of major-effect contributors identifies additional quantitative trait loci conditioning high-temperature growth in yeast. Genetics. 2008:180:1661-70.

11. Shapira R, David L. Genes with a combination of over-dominant and epistatic effects underlie heterosis in growth of Saccharomyces cerevisiae at high temperature. Front Genet. 2016;7:72.

12. Ben-Ari G, Zenvirth D, Sherman A, David L, Klutstein M, Lavi U, et al. Four linked genes participate in controlling sporulation efficiency in budding yeast. PLoS Genet. 2006;2:1815-23.

13. Deutschbauer AM, Davis RW. Quantitative trait loci mapped to singlenucleotide resolution in yeast. Nat Genet. 2005;37:1333-40.

14. Ehrenreich IM, Gerke JP, Kruglyak L. Genetic dissection of complex traits in yeast: Insights from studies of gene expression and other phenotypes in the BYxRM cross. Cold Spring Harb Symp Quant Biol. 2009;74:145-53.

15. Katou T, Namise M, Kitagaki H, Akao T, Shimoi H. QTL mapping of sake brewing characteristics of yeast. J Biosci Bioeng. 2009;107:383-93.

16. Nogami S, Ohya Y, Yvert G. Genetic complexity and quantitative trait loci mapping of yeast morphological traits. PLoS Genet. 2007;3:0305-18.

17. Kim HS, Fay JC. Genetic variation in the cysteine biosynthesis pathway causes sensitivity to pharmacological compounds. Proc Natl Acad Sci U S A. 2007;104:19387-91.

18. Voordeckers K, Kominek J, Das A, Espinosa-Cantú A, De Maeyer D, Arslan A, et al. Adaptation to high ethanol reveals complex evolutionary pathways. PLoS Genet. 2015;11:e1005635.

19. Greetham D, Wimalasena TT, Leung K, Marvin ME, Chandelia Y, Hart AJ, et al. The genetic basis of variation in clean lineages of Saccharomyces cerevisiae in response to stresses encountered during bioethanol fermentations. PLoS One. 2014;9:e103233.

20. Albert FW, Treusch S, Shockley AH, Bloom JS, Kruglyak L. Genetics of single-cel protein abundance variation in large yeast populations. Nature. 2014;506:1-19.
21. Parts L, Liu Y-C, Tekkedil MM, Steinmetz LM, Caudy AA, Fraser AG, et al. Heritability and genetic basis of protein level variation in an outbred population. Genome Res. 2014;24:1363-70.

22. Brauer MJ, Christianson CM, Pai DA, Dunham MJ. Mapping novel traits by array-assisted bulk segregant analysis in Saccharomyces cerevisiae. Genetics. 2006;173:1813-6.

23. Li J, Wang L, Wu X, Fang O, Wang L, Lu C, et al. Polygenic molecular architecture underlying non-sexual cell aggregation in budding yeast. DNA Res. 2013;20:55-66.

24. Marullo P, Bely M, Masneuf-Pomarède I, Pons M, Aigle M, Dubourdieu D. Breeding strategies for combining fermentative qualities and reducing offflavor production in a wine yeast model. FEMS Yeast Res. 2006;6:268-79.

25. Ambroset C, Petit M, Brion C, Sanchez I, Delobel P, Guérin C, et al. Deciphering the molecular basis of wine yeast fermentation traits using a combined genetic and genomic approach. G3Genes|Genomes|Genetics. 2011;1:263-81

26. Salinas F, Cubillos FA, Soto D, Garcia V, Bergström A, Warringer J, et al. The genetic basis of natural variation in oenological traits in Saccharomyces cerevisiae. PLoS One. 2012;7:e49640.

27. García-Ríos E, López-Malo M, Guillamón JM. Global phenotypic and genomic comparison of two Saccharomyces cerevisiae wine strains reveals a novel role of the sulfur assimilation pathway in adaptation at low temperature fermentations. BMC Genomics. 2014;15:1059.

28. Liti G, Haricharan S, Cubillos FA, Tierney AL, Sharp S, Bertuch AA, et al. Segregating YKU80 and TLC1 alleles underlying natural variation in telomere properties in wild yeast. PLoS Genet. 2009;5:e1000659.

29. Ramazzotti M, Berná L, Stefanini I, Cavalieri D. A computational pipeline to discover highly phylogenetically informative genes in sequenced genomes: application to Saccharomyces cerevisiae natural strains. Nucleic Acids Res. 2012:40:3834-48.

30. Liti G, Carter DM, Moses AM, Warringer J, Parts L, James SA, et al. Population genomics of domestic and wild yeasts. Nature. 2009;458:337-41.

31. Bergström A, Simpson JT, Salinas F, Barré B, Parts L, Zia A, et al. A highdefinition view of functional genetic variation from natural yeast genomes. Mol Biol Evol. 2014;31:872-88.

32. Liti G, Louis EJ. Advances in quantitative trait analysis in yeast. PLoS Genet. 2012;8:e1002912.

33. Naithani S, Saracco SA, Butler CA, Fox TD. Interactions among COX1, COX2, and COX3 mRNA-specific translational activator proteins on the inner surface of the mitochondrial inner membrane of Saccharomyces cerevisiae. Mol Biol Cell. 2003;14:324-33.

34. Ashbysb MN, Kutsunais SY, Ackermany S, Tzagoloffll A, Edwards PA. COQ2 is a candidate for the structural gene encoding puru-hydroxybenzoate: polyprenyltransferase. J Biol Chem. 1992;267:4128-36.

35. Kim HS, Huh J, Riles L, Reyes A, Fay JC. A noncomplementation screen for quantitative trait alleles in Saccharomyces cerevisiae. G3 (Bethesda). 2012:2:753-60

36. Lendenmann MH, Croll D, Palma-Guerrero J, Stewart EL, McDonald BA. QTL mapping of temperature sensitivity reveals candidate genes for thermal adaptation and growth morphology in the plant pathogenic fungus Zymoseptoria tritici. Heredity (Edinb). 2016;116:384-94.

37. Parts L, Cubillos FA, Warringer J, Jain K, Salinas F, Bumpstead SJ, et al. Revealing the genetic structure of a trait by sequencing a population under selection. Genome Res. 2011;21:1131-8.

38. Ehrenreich IM, Torabi N, Jia Y, Kent J, Martis S, Shapiro JA, et al. Dissection of genetically complex traits with extremely large pools of yeast segregants. Nature. 2010;15:1030-42

39. Cubillos FA, Billi E, Zörgö E, Parts L, Fargier P, Omholt S, et al. Assessing the complex architecture of polygenic traits in diverged yeast populations. Mol Ecol. 2011;20:1401-13.

40. Ames RM, Rash BM, Hentges KE, Robertson DL, Delneri D, Lovell SC. Gene duplication and environmental adaptation within yeast populations. Genome Biol Evol. 2010;2:591-601.

41. Brown CA, Murray AW, Verstrepen KJ. Rapid expansion and functional divergence of subtelomeric gene families in yeasts. Curr Biol. 2010;20:895-903.

42. Grant CM, Maclver FH, Dawes IW. Glutathione is an essential metabolite required for resistance to oxidative stress in the yeast Saccharomyces cerevisiae. FEBS Lett. 1997;29:511-5.

43. García-Ríos E, Ramos-Alonso L, Guillamón JM. Correlation between low temperature adaptation and oxidative stress in Saccharomyces cerevisiae. Front Microbiol. 2016;7:1-11. 
44. Beltran G, Novo M, Leberre V, Sokol S, Labourdette D, Guillamón JM, et al. Integration of transcriptomic and metabolic analyses for understanding the global responses of low-temperature winemaking fermentations. FEMS Yeast Res. 2006;6:1167-83.

45. Redón M, Guillamón JM, Mas A, Rozés N. Effect of growth temperature on yeast lipid composition and alcoholic fermentation at low temperature. Eur Food Res Technol. 2011;232:517-27.

46. Tronchoni J, Rozès N, Querol A, Guillamón JM. Lipid composition of wine strains of Saccharomyces kudriavzevii and Saccharomyces cerevisiae grown at low temperature. Int J Food Microbiol. 2012;155:191-8.

47. Henderson CM, Lozada-Contreras M, Jiranek V, Longo ML, Block DE. Ethanol production and maximum cell growth are highly correlated with membrane lipid composition during fermentation as determined by lipidomic analysis of 22 Saccharomyces cerevisiae strains. Appl Environ Microbiol. 2013:79:91-104.

48. Dawes IW, Hardie ID. Selective killing of vegetative cells in sporulated yeast cultures by exposure to diethyl ether. Mol Gen Genet. 1974;131:281-9.

49. Riou C, Nicaud JM, Barre P, Gaillardin C. Stationary-phase gene expression in Saccharomyces cerevisiae during wine fermentation. Yeast. 1997;13:903-15.

50. Zwietering MH, Jongenburger I, Rombouts FM, Van K. Modeling of the bacterial growth curve modeling of the bacterial growth curve. Appl Environ Microbiol. 1990;56:1875-81.

51. Deatherage DE, Barrick JE. Identification of mutations in laboratory-evolved microbes from next-generation sequencing data using breseq. Methods Mol Biol. 2014;1151:165-88.

52. Langmead B, Salzberg SL. Fast gapped-read alignment with Bowtie 2. Nat Methods. 2012;9:357-9.

53. Xie C, Tammi MT. CNV-seq, a new method to detect copy number variation using high-throughput sequencing. BMC Bioinformatics. 2009;10:80.

54. Katoh K, Standley DM. MAFFT multiple sequence alignment software version 7: improvements in performance and usability. Mol Biol Evol. 2013;30:772-80.

55. Stamatakis A. RAxML version 8: a tool for phylogenetic analysis and postanalysis of large phylogenies. Bioinformatics. 2014;30:1312-3.

56. Steinmetz LM, Sinha H, Richards DR, Spiegelman Jl, Oefner PJ, McCusker JH, et al. Dissecting the architecture of a quantitative trait locus in yeast. Nature. 2002;416:326-30.

57. Upshall A, Giddings B, Mortimore ID. The use of benlate for distinguishing between haploid and diploid strains of Aspergillus nidulans and Aspergillus terreus. J Gen Microbiol. 1977;100:413-8.

58. Stearns T, Hoyt MA, Botstein D. Yeast mutants sensitive to antimicrotubule drugs define three genes that affect microtubule function. Genetics. 1990;124:251-62.

59. Huxley C, Green ED, Dunham I. Rapid assessment of S. cerevisiae mating type by PCR. Trends Genet. 1990;6:236.

60. Brem RB, Kruglyak $L$. The landscape of genetic complexity across 5,700 gene expression traits in yeast. Proc Natl Acad Sci U S A. 2005;102:1572-7.

\section{Submit your next manuscript to BioMed Central and we will help you at every step:}

- We accept pre-submission inquiries

- Our selector tool helps you to find the most relevant journal

- We provide round the clock customer support

- Convenient online submission

- Thorough peer review

- Inclusion in PubMed and all major indexing services

- Maximum visibility for your research

Submit your manuscript at www.biomedcentral.com/submit

C) Biomed Central 